\title{
Rechazo parental y ajuste psicológico infantil: Efecto moderador del afecto familiar percibido desde una perspectiva multi-informante
}

\author{
Eva Izquierdo-Sotorrío ${ }^{1,2}$, Miguel Á. Carrasco ${ }^{2}$ y F. Pablo Holgado-Tello ${ }^{2}$ \\ ${ }^{1}$ Universidad a Distancia de Madrid, Madrid, España \\ ${ }^{2}$ Universidad Nacional de Educación a Distancia, Madrid, España
}

\begin{abstract}
Parental rejection and children's psychological adjustment: The moderating role of perceived family warmth
\end{abstract} from a multi-informant perspective.

\begin{abstract}
This study explores the moderating effect of children's perceptions about family environment on the relation between parental acceptance and children's psychological adjustment, from a multi-informant perspective. The sample consisted in 227 triads, father, mother and child from the same family (children's mean age 12.52, $S D=1.81$ ) divided in two groups based on children's level of perceived family warmth. Parental Acceptance-Rejection Questionnaire (PARQ) was used to assess parental acceptance-rejection, and CBCL (Child Behavioral Checklist) and YSR (Youth Self Report) from Achenbach System were used to assess children's psychological adjustment. Results showed that relations between parental rejection and adjustment depend on the informant used, and that the subjective perception of the child's contextual affection moderated the effect of maternal rejection on exteriorized problems informed by the father. The need to include children, mothers, and fathers in the assessment of family relations and children's psychological adjustment is highlighted.
\end{abstract}

Key words: Multi-informant; parental acceptance-rejection; mother; father: children psychological adjustment.

Resumen: El presente trabajo explora el efecto moderador de la percepción de niños y niñas sobre el ambiente familiar, desde una perspectiva multi-informante, en la relación entre rechazo parental y ajuste psicológico infantil. La muestra estuvo constituida por 227 triadas (padre, madre e hijo/a) de la misma familia (edad media de los menores 12.52, DT = 1.81). La aceptación-rechazo parental se evaluó mediante el PARQ (Parental Acceptance Rejection Questionnaire) y el ajuste mediante el CBCL (Child Behavioral Checklist) y el YSR (Youth Self-report). Los resultados mostraron diferencias en la asociación entre rechazo parental y ajuste condicionadas por la fuente informante, y que la percepción subjetiva del menor del afecto contextual moderaba el efecto del rechazo materno sobre los problemas exteriorizados informados por el padre. Se destaca la necesidad de incluir tanto a los propios menores como a sus padres y madres en la evaluación de las relaciones familiares y el ajuste psicológico infantil.

Palabras clave: Multi-informante; aceptación-rechazo parental; madre; padre; ajuste psicológico infantil.

\section{Introducción}

El ajuste psicológico infantil tiene su mayor exponente en el grado de adaptación que alcanzan los menores en

Recibido: 05 de marzo 2020; aceptado: 15 de julio 2020

Correspondencia: Eva Izquierdo-Sotorrío, Universidad a Distancia de Madrid (UDIMA); Vía de Servicio A-6, 15, 28400 Collado Villalba, Madrid. Correo-e: eva.izq@cop.es el contexto en el que se desarrollan (Ordóñez-López, 2015). En el campo de la psicopatología infantil, dos han sido los grupos de indicadores con los que habitualmente se ha identificado el grado de desajuste psicológico: por una parte, la sintomatología exteriorizada, relativa a los indicadores externamente manifiestos tales como problemas de conducta, comportamientos delictivos, agresividad o hiperactividad; y, por otra, la sintomatología interiorizada, relativa a indicadores menos explícitos y manifiestamente privados o internos, que incluyen pro- 
blemas de ansiedad, depresión o somatización (Achenbach, 2017; Sánchez-Sánchez, Fernández-Pinto, Santamaría, Carrasco y del Barrio, 2016).

En el marco de la teoría de la aceptación-rechazo interpersonal (IPARTheory; previamente denominada teoría de aceptación-rechazo parental, PARTheory, Rohner, 2016) la evidencia acumulada, a lo largo de más de treinta años de investigación, ha mostrado consistentemente que la aceptación-rechazo parental es una de las variables más estrechamente asociadas al ajuste psicológico de los hijos e hijas en el contexto familiar. Desde esta perspectiva, la dimensión aceptación-rechazo se refiere a la calidad de los lazos afectivos que los hijos e hijas perciben de sus padres y madres como figuras significativas (Rodríguez, 2017). Multitud de estudios transculturales (Ali, Khaleque y Rohner, 2015; Carrasco, Delgado y Holgado-Tello, 2019) han señalado que la aceptación percibida por parte de las figuras relevantes en la vida de una persona incide en un desarrollo psicológico más favorable independientemente de su origen cultural, étnico o religioso. Sentirse aceptado (y no rechazado) por parte de las figuras parentales parece tener un gran impacto en el desarrollo de conductas y emociones saludables a lo largo de la vida (de Albéniz-Garrote, Rubio-Rubio y Medina-Gómez, 2018; Khaleque, 2018; Rodríguez, 2017).

La evaluación del rechazo parental se ha realizado, principalmente, mediante cuestionarios, bien informados por los hijos/as (autoinformes) bien por los padres o madres (heteroinformes). Sin embargo, la evaluación basada en la evidencia ha mostrado la conveniencia de emplear, en la evaluación infantil especialmente, una aproximación multidimensional, multimétodo y multifuente (Achenbach, 2017; De Los Reyes et al., 2019; Hunsley y Mash, 2007; Kazdin, 2005), la cual ha sido escasamente utilizada hasta la fecha en la contrastación de los postulados de la IPARTheory. Esta nueva aproximación a la evaluación de las relaciones entre aceptación-rechazo parental y ajuste psicológico de los hijos/ as permitiría obtener información desde la perspectiva de varios informantes e integrar la información inconsistente proporcionada por las diferentes fuentes y procedimientos de evaluación (Izquierdo-Sotorrío, Holgado-Te1lo y Carrasco, 2016). En este sentido, el uso de distintas fuentes informantes posibilita detectar variaciones en las manifestaciones de los niños y niñas en sus diversos contextos de socialización (Achenbach, Ivanova y Rescorla, 2017), identificar diferentes percepciones sobre los constructos evaluados (De Los Reyes et al., 2019) así como dar cuenta de los posibles errores de medida (Achenbach, 2017). Además, la comparación de la información aportada por las distintas fuentes permitirá, por una parte, estudiar en qué medida la perspectiva de uno de los informantes pudiera moderar la relación entre las variables en estudio (Schoppe-Sullivan y Fagan, 2020); y por otra, cómo la elección de unas fuentes informantes frente a otras o la combinación particular de unas y otras pudiera contribuir a la validez incremental de la información perseguida (Izquierdo-Sotorrío et al., 2016). Todo ello redundaría en una mejora de la predicción de las variables objeto de estudio, así como en la optimización del uso de los informantes para la toma de decisiones en el proceso de evaluación (Johnston y Murray, 2003).

Dentro del estudio de la relación entre ajuste psicológico y las relaciones familiares, es habitual el uso de instrumentos que tienen versiones para distintos informantes (madres/padres y menores, principalmente) (Achenbach et al., 2017; De Los Reyes et al., 2019). Si bien los instrumentos desarrollados por la IPARTheory permiten recopilar información de distintas fuentes (madre, padre y menor) en relación con la aceptación-rechazo de los hijos (paterna o materna) (Putnick et al., 2015; Rohner y Ali, 2016) y a pesar de la multitud de investigaciones que han apoyado la relación entre rechazo parental y ajuste psicológico infantil (Ali et al., 2015; Rodríguez et al., 2017) muy pocas han abordado su estudio desde una perspectiva multi-informante y menos aún han explorado el efecto moderador que la propia experiencia de aceptación-rechazo del menor tiene en la predicción del ajuste psicológico infantil a partir de la aceptación-rechazo parental.

Hasta donde nos consta, solo dos estudios previos (Khaleque, Rohner y Nahar, 2008; Rohner et al., 2005) han indagado las diferencias encontradas entre familias afectuosas y no afectuosas según la percepción autoinformada del menor. En ambas investigaciones, tras el análisis de las divergencias entre madre e hijos/as sobre rechazo parental, encuentran que la discrepancia entre fuentes tiende a aumentar en el grupo donde el menor percibe menos afecto.

Más allá de estos trabajos, no hemos hallado hasta la fecha, ninguna investigación que haya analizado el efecto moderador que dicha percepción del menor tiene sobre la relación entre aceptación-rechazo parental y ajuste psicológico infantil. No obstante, existen en la literatura algunos estudios que apuntan que la percepción de niños y niñas sobre la aceptación-rechazo familiar podrían moderar la relación entre otras conductas parentales y su propio ajuste psicológico. En este sentido, algunas investigaciones (Germán, Gonzales, McClain, Dumka y Millsap, 2013; Glicklich, 2014) han encontrado que la relación entre prácticas parentales y problemas exteriorizados solo se constata en los hijos/as que perciben un nivel elevado de rechazo en su contexto familiar. De manera si- 
milar, otros estudios han señalado que la relación entre prácticas punitivas parentales y problemas de conducta $\mathrm{o}$ sintomatología interiorizada (McKee et al., 2007) se ve incrementada cuando los menores perciben un menor afecto familiar.

El objetivo del presente estudio es doble. En primer lugar, analizar las relaciones del rechazo parental (paterno y materno) percibido por los padres/madres sobre los problemas de ajuste infantil (exteriorizados e interiorizados) dentro de dos contextos familiares diferentes: un contexto familiar percibido por los hijos como afectuoso y otro percibido como menos afectuoso; y en segundo lugar, analizar los efectos parciales del rechazo materno y paterno sobre el ajuste psicológico de los hijos, así como el efecto moderador que tiene la experiencia subjetiva de afecto familiar del propio menor en la relación entre el rechazo parental y los problemas de ajuste psicológico infantil.

De acuerdo con la literatura revisada y los objetivos previos establecidos, se plantean tres hipótesis: (1) en relación con el primer objetivo, esperamos que la correlación entre rechazo parental y ajuste psicológico en los hijos/as será significativamente mayor en el grupo de menores que perciban menos afecto familiar; en relación con el segundo objetivo se plantean la segunda y tercera hipótesis, (2) esperamos que tanto el rechazo materno como el paterno tendrán un efecto significativo y negativo sobre el ajuste psicológico de los menores; y (3) existirá un efecto moderador del afecto familiar percibido por el/la menor en la contribución del rechazo materno y paterno sobre los problemas exteriorizados e interiorizados de los hijos/as.

\section{Método}

\section{Participantes}

La muestra estuvo compuesta por 227 triadas (padre, madre e hijo/a) procedentes de la misma familia. Los menores $(60 \%$ chicas $)$ tenían edades comprendidas entre los 9 y los 17 años $(M=12.52, D T=1.81)$. Todos los menores estaban escolarizados y la mayoría vivían en hogares con dos figuras parentales (91\%). El 88\% de los padres y el $70 \%$ de las madres tenían empleo. El nivel educativo de los padres y madres era, respectivamente: estudios universitarios (40 y 35\%), educación secundaria $(40$ y $57 \%$ ) y primaria (20 y $8 \%$ ).

La muestra de estudio se extrajo de una más amplia procedente de una investigación diseñada para el análisis de la aceptación-rechazo parental y el ajuste psicológico infantil en la población española. Se trata de una muestra incidental obtenida de la selección de aquellos participantes que contaban con datos procedentes de su tríada correspondiente (madre-padre-menor), lo que representó el $22 \%$ de la muestra total de origen $(N=1036$ menores). La muestra de origen se seleccionó por muestreo aleatorio de escuelas públicas y concertadas en distintas regiones de España. La ratio de participación de las familias fue del $91 \%$. No se encontraron diferencias demográficas significativas (edad o sexo de los menores y nivel socioeconómico) entre las familias participantes y las no participantes.

Para los fines de esta investigación los participantes se distribuyeron en dos grupos según su nivel de afecto familiar percibido por el menor: Grupo $1(\mathrm{G} 1)=$ «Afecto» formado por los participantes cuyos niveles de afecto percibido se situaban por debajo de la media más una desviación tipo en la variable aceptación-rechazo parental autoinformado (padre, madre, o padre y madre); y Grupo 2 (G2) = «No-afecto», formado por los participantes cuyos niveles de afecto percibido se situaban por encima de la media más una desviación tipo en la variable aceptación-rechazo parental autoinformado (padre, madre, o padre y madre). Por lo tanto, el grupo 1 (afecto) se compone de aquellos menores que perciben un contexto familiar más afectuoso frente al grupo 2 (no-afecto) que perciben un contexto familiar menos afectuoso.

\section{Instrumentos}

Todos los instrumentos descritos fueron cumplimentados por los tres miembros de la tríada (padre, madre y menor) en sus versiones específicas.

Parental Acceptance-Rejection/Control Questionnaire (Child PARQ/Control; Rohner y Ali, 2016). Se utilizaron cuatro formas paralelas, dos para los niños/as, versión sobre el padre y versión sobre la madre; y dos para los padres, versión para la madre sobre sí misma y versión para el padre sobre sí mismo; en estas dos últimas versiones se utilizó la adaptación española de del Barrio, Ramírez-Uclés, Romero y Carrasco (2014). Este cuestionario consta de 29 ítems y es un autoinforme. Las distintas versiones son idénticas, la única diferencia es que están formuladas según la perspectiva de cada informante. El cuestionario contempla las cuatro posibles dimensiones de la variable aceptación-rechazo propuesta por la IPARTheory: (1) Calor/Afecto, (2) Hostilidad/Agresión, (3) Indiferencia/Negligencia y, (4) Rechazo indiferenciado. Existe una última dimensión (5) Control la cual fue excluida en este estudio. Consideradas conjuntamente las cuatro primeras componen una medida agregada de la percepción que tienen los hijos de la aceptación-rechazo procedente de sus padres. El cuestionario se contesta con una escala tipo Likert de 4 puntos: 4 
«casi siempre verdad», 3 «muchas veces verdad», 2 «a veces verdad» y 1 «casi nunca verdad». El rango de puntuaciones totales oscila de 24 a 96, donde el mínimo indica la mayor percepción de aceptación y el valor más alto supone la máxima percepción de rechazo. El punto de corte se establece en 60 , valores por debajo de dicho punto indican una percepción mayormente de aceptación por parte de las figuras parentales y, entre 60 y 96 mayormente de rechazo. Este cuestionario ha demostrado tener unas propiedades psicométricas excelentes (Rohner y Ali, 2016). La consistencia interna para cada una de las versiones del instrumento en esta muestra fue: .88 y .97 en las versiones de las menores referidas a padres y madres, respectivamente; y .88 y .88 en ambas versiones de padres y madres.

Inventario Autoaplicado de Problemas de Conducta para Jóvenes (YSR/6-18 años; Achenbach y Rescorla, 2001) y Children Behavioral Checklist (CBCL/4-18; Achenbach y Rescorla, 2001). Se utilizó la traducción de la Unitat d'Epidemiologia i de Diagnòstic en Psicopatologia del Desenvolupament, Universitat Autónoma de Barcelona. El YSR consta de dos partes: una primera, que no se utilizó en este estudio, que consta de 17 ítems y evalúa la adaptación social mediante tres subescalas que abordan aspectos deportivos, académicos y sociales, y una segunda, con 112 ítems, formulados en primera persona en referencia al presente y los últimos seis meses, que recoge un amplio número de problemas de conducta tanto interiorizados (ansiedad/depresión, retraimiento, quejas somáticas) como exteriorizados (ruptura de normas, conducta agresiva). Cada ítem se valora en una escala Likert de 3 puntos: 0 «No es cierto», 1 «Algo, algunas veces cierto», 3 «Cierto muy a menudo o bastante a menudo». Puntuaciones altas indican un mayor grado de desajuste conductual. Para el presente trabajo se utilizaron las escalas de problemas interiorizados y exteriorizados. El Children's Behavioral Check List (CBCL) es similar al YSR a excepción de que tiene un ítem más (113, «otros problemas»), se utilizaron las mismas escalas que en el YSR. Ambas escalas muestran propiedades psicométricas adecuadas (Achenbach, 2017). Para la muestra de este estudio, la consistencia interna fue de .75 y .73 para las escalas exteriorizadas e interiorizadas del YSR; .80 y. 79 para dichas escalas del CBCL del padre y .77 y .78 para el CBCL de la madre.

\section{Procedimiento}

Una vez que la muestra fue seleccionada, se solicitó consentimiento tanto a los colegios participantes como a padres/madres o tutores legales de los menores que formaron parte de la investigación. Por tanto, la participa- ción fue voluntaria previo consentimiento. Los niños y niñas cumplimentaron los instrumentos colectivamente durante la jornada escolar en dos ocasiones distintas, antes y después del recreo. Las sesiones duraron, aproximadamente, 45 minutos. Durante la primera se contestó al cuestionario sociodemográfico y el PARQ y durante la segunda el PARQ y el YSR. Al final de esta sesión se entregaban a cada menor los cuestionarios que debían cumplimentar tanto sus padres como sus madres (PARQ-C y el CBCL) con la consigna de que debían ser devueltos en un plazo máximo de una semana. Los cuestionarios cumplimentados por los padres/madres eran devueltos por los menores a sus profesores quienes lo hacían llegar a los investigadores en sobre cerrado en el que constaba el número de identificación del participante.

La investigación fue previamente aprobada por el Comité bioético de la Universidad Nacional de Educación a Distancia.

\section{Análisis estadísticos y variables}

En primer lugar, se realizó un análisis exploratorio preliminar mediante correlaciones bivariadas y parciales con el fin de indagar la relación entre variables en cada uno de los grupos (G1 = Afecto vs. G2 = No-afecto $)$. Posteriormente, se realizaron seis regresiones jerárquicas tomando como variables independientes la aceptación-rechazo materna y paterna informadas por las madres/padres y como variables criterio las dimensiones globales exteriorizadas e interiorizadas para cada uno de los tres informantes; el nivel de afecto familiar se consideró como variable moderadora. Para estos análisis se introdujo la aceptación-rechazo paterna junto con la aceptación-rechazo materna en el primer paso y, en el segundo paso, el producto de la aceptación-rechazo materna y paterna por la variable «Grupo de afecto» con el fin de explorar posibles interacciones. Finalmente, se realizó un análisis post-hoc de las interacciones significativas obtenidas en las regresiones previas mediante una aproximación de punto de corte a partir del que se establecieron los grupos.

Los datos se analizaron utilizando SPSS versión 20.0 para Windows con el comando PROCESS para el análisis de las interacciones, junto con el paquete estadístico R.

\section{Resultados}

\section{Análisis preliminares}

En la figura 1 se muestra la distribución de los dos grupos de participantes establecidos. La comparación de 
grupos por edad (media) no muestra diferencias significativas $(p<.05)$, aunque se aprecia un incremento tendencial de la edad en el grupo de no-afecto. La distribución por sexo fue similar en ambos grupos.

Figura 1. Distribución muestral según la agrupación de los participantes en afecto y no-afecto

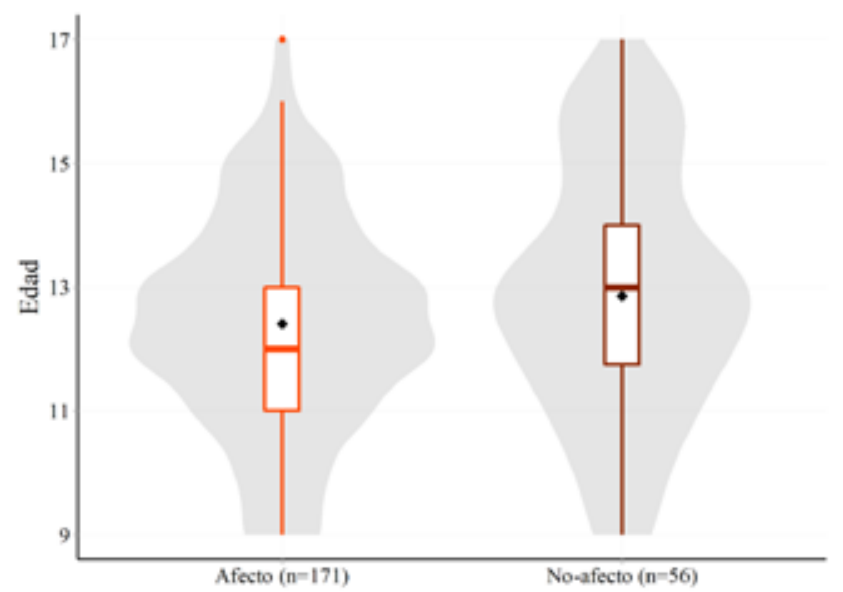

Nota $:=$ Media

En la Tabla 1 se muestran las correlaciones, por grupo de afecto vs. no-afecto, entre aceptación-rechazo materna y paterna y los problemas exteriorizados e interiorizados informados por las distintas fuentes: padre, madre y menor. En el caso de los problemas exteriorizados informados por los padres y madres, solo algunas correlaciones con el rechazo parental resultan significativas. Entre ellas cabe destacar la correlación entre el rechazo materno y los problemas exteriorizados informados por el padre en el grupo de no-afecto. No aparecen correlaciones significativas entre los problemas interiorizados y el rechazo parental cuando son los padres o madres quienes informan sobre dichos problemas. Sin embargo, cuando el informante del ajuste es el menor, todas las correlaciones resultan significativas, siendo de nuevo la más elevada entre rechazo materno y problemas exteriorizados en el grupo de no-afecto. En todos los casos, las correlaciones resultan positivas y tienden a mostrar mayor relación en el grupo de no-afecto, lo que indica que a mayor percepción de rechazo parental mayor desajuste infantil especialmente en el grupo de menores que perciben menos afecto familiar.

Con la finalidad de comparar las correlaciones entre ambos grupos se utilizó el estadístico de contraste $Z$ de Fisher (1925). El análisis de las diferencias entre correlaciones indicó solo una diferencia significativa $(z=-2.7$, $p=.01)$ para la correlación entre la aceptación-rechazo materna y los problemas exteriorizados informados por el padre. Esta correlación mostró un valor significativamente superior en el grupo de no-afecto $\left(.41^{* *}\right)$ frente al de afecto (.01). Asimismo, la diferencia de medias entre grupos para el rechazo paterno $\left(t_{(71)}=-2.93, p=.01\right) \mathrm{y}$ para la materno $\left(t_{(74)}=-3.22, p=.00\right)$ resultaron significativas, lo que indica que en el grupo de no-afecto familiar, tanto el rechazo paterno y materno informados por padre y madre respectivamente fueron significativamen-

Tabla 1. Correlaciones entre la aceptación-rechazo parental y el ajuste psicológico del menor en los grupos de afecto y no afecto

\begin{tabular}{|c|c|c|c|c|c|c|c|c|}
\hline & \multicolumn{2}{|c|}{ Afecto } & \multicolumn{6}{|c|}{ No-afecto } \\
\hline & PARQP & PARQM & Media & $\mathrm{Dt}$ & PARQP & PARQM & Media & Dt \\
\hline Prob. Ext. Inf: P & $.19^{*}$ & .01 & 3.79 & 3.19 & $.30 *$ & $.41 * *$ & 7.37 & 6.56 \\
\hline Prob. Int. Inf: P & .11 & .02 & 4.76 & 4.36 & .21 & .21 & 7.48 & 6.40 \\
\hline Prob. Ext. Inf: M & $.28 * *$ & .15 & 4.17 & 3.77 & .20 & $.26^{*}$ & 7.89 & 6.45 \\
\hline Prob. Int. Inf: M & .11 & .10 & 5.36 & 5 & .23 & .11 & 9.16 & 6.14 \\
\hline Prob. Ext. Inf: $\mathrm{N}$ & $.38 * *$ & $.40 * *$ & 12.8 & 9.06 & $.38 * *$ & $.50 * *$ & 15.42 & 11.11 \\
\hline Prob. Int. Inf: $\mathrm{N}$ & $.21 * *$ & $.16^{*}$ & 16.63 & 9.96 & $.23^{*}$ & $.19^{*}$ & 19.57 & 11.39 \\
\hline Media & 34.33 & 31.74 & - & - & 39.04 & 36.56 & - & - \\
\hline Desviación tipo & 7.4 & 7.36 & - & - & 11.25 & 10.37 & - & - \\
\hline
\end{tabular}

Nota . Prob. Ext = problemas exteriorizados; Prob. Int = Problemas interiorizados; Inf: $\mathrm{P}=$ Informante padre; Inf: $\mathrm{M}=$ Informante madre; Inf: $\mathrm{N}=$ Informante niño/a; PARQP = Aceptación-rechazo paternal informada por el padre, PARQM = Aceptación-rechazo maternal informada por la madre $*_{p}<.05, * * p<.01$. 
te más elevados que en el grupo de afecto, lo que viene a corroborar que existe un grado elevado de congruencia entre la percepción de rechazo del menor y la de los padres/madres.

Posteriormente a estos análisis, se realizaron correlaciones parciales en ambos grupos (afecto y no-afecto) entre las variables con el objetivo de controlar el efecto del rechazo por parte de un padre/madre sobre la correlación entre el rechazo del otro padre/madre y el ajuste. Como se puede apreciar en la Tabla 2, al controlar el rechazo del otro padre o madre, en el grupo de afecto se mantiene significativa la relación paterna con los problemas exteriorizados independientemente de quién informe de esta última variable. En este mismo grupo, el descenso respecto de los valores de las correlaciones bivariadas, es más acusado en el rechazo materno (al controlar el paterno), cuya relación solo es significativa cuando es el menor el informante de los síntomas exteriorizados. Por lo tanto, en el grupo de afecto parece tener mayor relevancia el rechazo paterno. Sin embargo, en el grupo de no-afecto el rechazo materno es el único que sigue siendo significativo en relación con los problemas exteriorizados informados por padre y menor, por lo que en este grupo el rechazo materno parece jugar un papel más relevante.

Efectos parciales del rechazo parental y efecto moderador del afecto familiar (percepción del menor) sobre la relación entre el rechazo parental y los problemas de ajuste psicológico infantil

Se llevaron a cabo seis regresiones jerárquicas (Tabla 3 ), una por cada variable dependiente. Las variables in- dependientes (aceptación-rechazo paterna y aceptación-rechazo materna) se introdujeron en el paso 1, con el fin de analizar sus efectos directos parciales. En el segundo paso se introdujeron los productos del afecto familiar (en términos de percepción del menor) por la aceptación-rechazo materno y paterno con el objetivo de analizar el efecto moderador del grupo de pertenencia. La aceptación-rechazo paterna mostró efectos directos significativos y positivos sobre el ajuste psicológico infantil (paso 1) independientemente del informante del ajuste. Sin embargo, la aceptación-rechazo materna solo resultó significativa, y positiva, cuando el menor informaba de problemas exteriorizados. Finalmente, dos de las interacciones resultaron significativas (paso 2). El rechazo materno por el afecto familiar cuando el padre informaba de los problemas exteriorizados (Prob. Ext. Inf: $\mathrm{P}: \beta=1.01, t=3.00, p=.01)$ y cuando era la madre la que informaba de esta misma dimensión del ajuste (Prob. Ext. Inf: M: $\beta=.77, t=2.23, p=.03$ ).

En el análisis post hoc de las interacciones, solo resultó significativa la moderación del afecto familiar percibido por el menor sobre la relación entre rechazo materno y problemas exteriorizados de los hijos/as informados por el padre. Tal y como se muestra en la figura 2 , en el Grupo de no-afecto el efecto del rechazo materno sobre los problemas exteriorizados del menor $(\beta=.26$, $p=.00)$ es significativamente superior que en el Grupo de afecto $(\beta=.00, p=.93)$. En el grupo de «afecto» el efecto no es significativo. Estos resultados sugieren un efecto intensificador del contexto familiar no afectuoso sobre el rechazo materno (no paterno) en el ajuste psicológico de los hijos/as informado por el padre.

Tabla 2. Correlaciones parciales entre los problemas de ajuste psicológico y la aceptación-rechazo parental en el grupo de afecto versus no-afecto (variable de control aceptación-rechazo materno y paterno, respectivamente)

\begin{tabular}{lcccc}
\hline & Afecto & & No- afecto \\
& PARQP & PARQM & PARQP & .14 \\
Prob. Ext. Inf: P & $.23^{* *}$ & -.13 & .13 & .13 \\
Prob. Int. Inf: P & .12 & -.06 & .09 & .20 \\
Prob. Ext. Inf: M & $.25^{* *}$ & -.03 & .20 & .01 \\
Prob. Int. Inf: M & .07 & .04 & .19 & $.39 * *$ \\
Prob. Ext. Inf: N & $.19^{*}$ & $.24 * *$ & .16 \\
Prob. Int. Inf: N & .14 & .05 & .09 \\
\hline
\end{tabular}

Nota . Prob. Ext = problemas exteriorizados; Prob. Int = Problemas interiorizados; Inf: $\mathrm{P}=$ Informante padre; Inf: $\mathrm{M}=$ Informante madre; Inf: $\mathrm{N}=$ Informante niño/a; PARQP = Aceptación-rechazo paternal informada por el padre, $\mathrm{PARQM}=$ Aceptación-rechazo maternal informada por la madre.

$* p<.05, * * p<.01$. 
Tabla 3. Análisis de regresión jerárquica de la predicción del ajuste psicológico infantil

\begin{tabular}{|c|c|c|c|c|c|c|c|c|c|c|c|c|}
\hline \multirow[t]{2}{*}{ Predictores } & \multicolumn{2}{|c|}{ Prob. Ext. Inf: P } & \multicolumn{2}{|c|}{ Prob. Int. Inf: P } & \multicolumn{2}{|c|}{ Prob. Ext. Inf: M } & \multicolumn{2}{|c|}{ Prob. Int. Inf: M } & \multicolumn{2}{|c|}{ Prob. Ext. Inf: N } & \multicolumn{2}{|c|}{ Prob. Int. Inf: N } \\
\hline & $\beta$ & $R^{2} / D R^{2}$ & $\beta$ & $R^{2} / D R^{2}$ & $\beta$ & $R^{2} / D R^{2}$ & $\beta$ & $R^{2} / D R^{2}$ & $\beta$ & $R^{2} / D R^{2}$ & $\beta$ & $R^{2} / D R^{2}$ \\
\hline \multicolumn{13}{|l|}{ Paso 1} \\
\hline PARQP & $.23^{* *}$ & $R^{2}=.01$ & $.17^{*}$ & $R^{2}=.03$ & $.23 * *$ & $R^{2}=.10$ & $.17^{*}$ & $R^{2}=-.04$ & $.20^{* *}$ & $R^{2}=.22$ & $.18^{*}$ & $R^{2}=.05$ \\
\hline PARQM & .13 & $D R^{2}=.11^{* *}$ & .05 & $D R^{2}=.04 * *$ & .13 & $D R^{2}=.10^{* *}$ & .07 & $D R^{2}=.05 * *$ & $.33^{* *}$ & $D R^{2}=.23 * *$ & .09 & $D R^{2}=.06^{* *}$ \\
\hline \multicolumn{13}{|l|}{ Paso 2} \\
\hline PARQM X Afecto & $1.01^{* *}$ & $R^{2}=.20$ & .44 & $R^{2}=-.07$ & $.77^{*}$ & $R^{2}=.17$ & -.02 & $R^{2}=.09$ & .20 & $R^{2}=.22$ & .12 & $R^{2}=.05$ \\
\hline PARQP X Afecto & -.57 & $D R^{2}=.11^{* *}$ & -15 & $D R^{2}=.04 * *$ & -.38 & $D R^{2}=.08^{* *}$ & .38 & $D R^{2}=.06^{* *}$ & -.22 & $D R^{2}=.00$ & -.04 & $D R^{2}=.00$ \\
\hline
\end{tabular}

Nota . Prob. Ext = problemas exteriorizados; Prob. Int = Problemas interiorizados; Inf: $\mathrm{P}=$ Informante padre; Inf: $\mathrm{M}=$ Informante madre; Inf: $\mathrm{N}=$ Informante niño/a; PARQP = Aceptación-rechazo paternal informada por el padre, PARQM = Aceptación-rechazo maternal informada por la madre.

$* p<.05, * * p<.01$.

Figura 2. Moderación del afecto familiar percibido por el menor sobre la relación entre el rechazo materno y los problemas exteriorizados

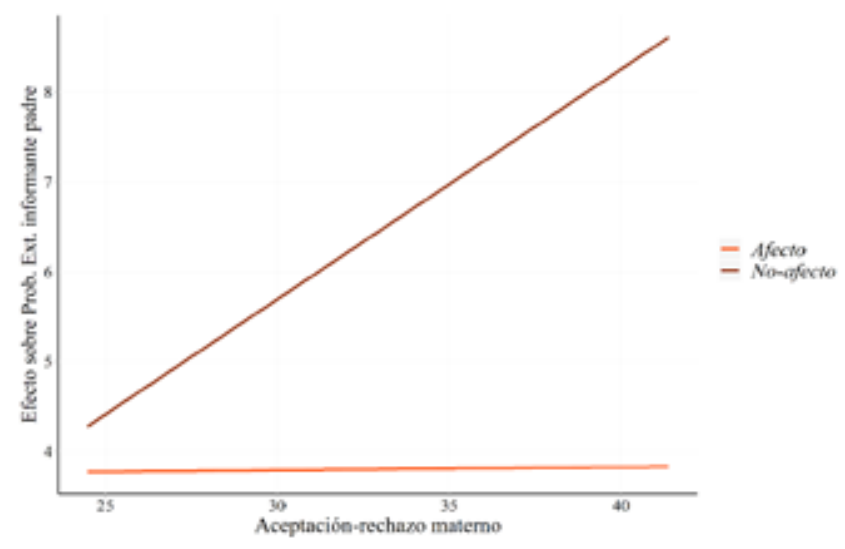

Discusión

El objetivo de este estudio era, por una parte, analizar la relación entre rechazo parental (paterno y materno) y ajuste infantil (problemas exteriorizados e interiorizados) dentro de dos contextos familiares diferentes: un contexto familiar percibido por los hijos/as como afectuoso y otro percibido como menos afectuoso; y por otra, analizar el efecto moderador del afecto familiar (percepción del menor) sobre la predicción de los problemas de ajuste psicológico infantil a partir del rechazo parental, y todo esto desde una perspectiva multi-informante.

Muy pocos estudios han abordado la relación entre ajuste psicológico infantil y rechazo parental desde una perspectiva multi-informante $\mathrm{y}$, hasta donde sabemos, este es el primer estudio que indaga sobre el efecto moderador de la propia experiencia de aceptación-rechazo del menor, por lo que estos datos son una importante contribución al conocimiento sobre la predicción del ajuste a partir de la aceptación-rechazo parental y a la optimización del uso de los informantes para la toma de decisiones en el proceso de evaluación.

Tal y como esperábamos, los resultados de esta investigación han mostrado que el rechazo parental se asocia significativamente con un incremento de los problemas de ajuste en los menores, no obstante, estas relaciones parecen estar condicionadas tanto por la fuente informante como por el contexto familiar de afecto percibido por los hijos/as. Más específicamente, la relación entre rechazo parental y ajuste tendía a ser más robusta en el grupo de menores que percibían menos afecto familiar (hipótesis $1^{\text {a }}$ ) $\sin$ embargo esto estaba condicionado por la fuente informante: en ambos grupos, afecto vs. no-afecto, los problemas exteriorizados e interiorizados se asocian significativamente al rechazo materno y/o paterno cuando informa el menor de los problemas de ajuste. Estas relaciones, en cambio, no son homogéneas cuando los problemas de ajuste son informados por los padres o las madres: el rechazo materno no se asocia con los problemas de ajuste en el grupo de afecto y solo lo hace con los exteriorizados en el grupo de no afecto; el rechazo paterno se asocia con los problemas exteriorizados en ambos grupos, excepto en el grupo de no afecto cuando estos problemas son informados por la madre. En ningún caso el rechazo parental se asoció con los problemas interiorizados de los hijos/as cuando eran los padres/madres quienes informaban de ello. Por tanto, la hipótesis primera se confirma parcialmente en la medida que su constatación no se da en todos los casos y parece depender de quién informe sobre los problemas de ajuste, así como del contexto familiar de afecto o no afecto percibido.

Como ha mostrado la literatura extensamente, el rechazo parental y los problemas interiorizados y exterio- 
rizados se asocian positiva y significativamente (Ali et al., 2015; Rodríguez, 2017; Rohner, 2004), lo que se ha mostrado consistente con estos resultados. No podemos afirmar lo mismo, con tanta rotundidad, cuando estas relaciones se estudian a la luz de las fuentes informantes o el afecto familiar percibido. El grado de significación de las relaciones, así como el valor absoluto de sus correlaciones parece modificarse según quien informe de la aceptación-rechazo parental o del ajuste de los hijos en un contexto de afecto familiar versus en un contexto de no afecto. No tenemos aún una explicación para dar cuenta de estas diferencias, no es posible comparar estos resultados con estudios previos dado que no hemos hallado investigaciones que hayan abordado la relación de estas variables desde una perspectiva multifuente. No obstante, la literatura indica que la variabilidad encontrada habitualmente entre distintos informantes puede deberse: al efecto del método de recogida de información (i.e. autoinformado vs. heteroinformado) (Neyer, 2006), a la accesibilidad a la información que cada fuente informante tiene según la naturaleza interna-privada versus externa-manifiesta del contenido a evaluar (Johnston y Murray, 2003; van Doorn et al., 2018), al mejor despliegue de ciertas conductas en unos contextos y no en otros (Achenbach et al., 2017; De Los Reyes et al., 2019), a la concepción de cada informante sobre lo que constituye un comportamiento normal (Richters, 1992), al propio estado emocional del informante (Berg-Nielsen, Vika y Dahl, 2003) y/o a la diferencia en la percepción de las prácticas parentales o las relaciones entre padres/madres e hijos/as (Hoeve et al., 2009).

La tendencia incrementada del valor absoluto de las correlaciones entre rechazo parental y desajuste psicológico que se ha hallado en este estudio es esperable, en parte, por el posible efecto sinérgico del ambiente familiar global de no-afecto y el rechazo parental, lo que además es congruente con estudios previos (Germán et al., 2013; Glicklich, 2014; McKee et al., 2007). No obstante, esta tendencia tampoco resultó homogénea en todos los supuestos y de nuevo parece estar condicionada tanto por la fuente informante como por la naturaleza exteriorizada o interiorizada de los problemas de ajuste. Las asociaciones entre rechazo materno o paterno y problemas interiorizados resultaron similares y no significativas tanto en el grupo de afecto como en el de no afecto; en cambio cuando se trataba de problemas exteriorizados, el rechazo paterno (no el materno) se asoció significativamente con estos problemas en el grupo de afecto y no afecto, a excepción de que fuera la madre quien informara sobre estos problemas, cuya relación no resultaba significativa en el grupo de no afecto; el rechazo materno solo se asoció con los problemas exteriorizados en el grupo de no afecto. Además, el rechazo tanto materno como paterno se asociaba significativamente con los problemas interiorizados y exteriorizados, cuando estos eran informados por los menores. La fuente niño es la que mayor coherencia ha mostrado en los resultados, como suele suceder en la mayoría de los trabajos.

No tenemos una explicación sobre las diferencias en estos patrones específicos de relaciones y solo nos queda constatarlas. Si bien, las relaciones significativas encontradas exclusivamente entre rechazo parental y los problemas interiorizados cuando es el menor quien informa del ajuste, está en consonancia con aquellas investigaciones (Johnston y Murray, 2003; van Doorn et al., 2018) que justifican estos resultados por la naturaleza interna y privada de los problemas interiorizados, a los cuales los menores tienen el mejor acceso. Son también consistentes con los estudios que los ha mostrado a los menores como los mejores informantes frente a los padres y madres cuando se pretende predecir el desajuste a partir del rechazo parental (Izquierdo-Sotorrío et al., 2016).

Por otra parte, es importante destacar que cuando las relaciones entre rechazo parental y ajuste de los hijos se analizan controlando el efecto del otro padre/madre, en el grupo de afecto es el rechazo paterno el que parece tener más relevancia mientras que en el grupo de no-afecto es el rechazo materno. Esto sugiere el posible efecto moderador del contexto de afecto familiar percibido por los hijos/as. Al controlar el efecto del rechazo parental de madre o padre en estas relaciones se observó que en los contextos familiares afectuosos (grupo donde la media global de rechazo percibido es menor) el rechazo materno y, especialmente, el paterno se asociaba significativamente con los problemas exteriorizados (no interiorizados). En cambio, en los contextos percibidos como no afectuosos, solo el rechazo de la madre (no del padre) se asociaba con los problemas exteriorizados del niño/a solo informados por el padre. La aceptación-rechazo paterna parece tener más relevancia cuando se trata de un grupo familiar afectuoso, en cambio es la aceptación-rechazo materna la que adquiere más relevancia en cuando se trata de un contexto familiar no afectuoso. Cummings et al. (2010) afirman que la menor delimitación del rol paterno frente al materno hace que la figura paterna sea más sensible a los contextos en los que ocurre, lo que podría explicar parte de nuestros resultados. En este sentido, el contexto percibido como menos afectuoso podría «debilitar» o aminorar el efecto del rechazo paterno. Es importante señalar, que el rechazo materno (una vez controlado el paterno) solo se asociaba significativamente con los problemas exteriorizados (no interiorizados) cuando los informa el propio niño. De nuevo no tenemos explicación para este resultado, aunque po- 
demos especular con la idea de que los problemas interiorizados aparecerían con el rechazo sumativo de ambos progenitores. Algunos estudios apoyan la idea de que es la combinación de la aceptación-rechazo de ambos padres en un efecto sinérgico (Meunier, Bisceglia y Jenkins, 2012) lo que predice el ajuste psicológico y, además, afirman que la madre es quien tiene un efecto más predominante. Este resultado se fundamenta, en parte, por la cultura patriarcal de nuestro contexto social en el que la madre es la que ostenta el rol principal en la crianza de los hijos/as. Por otro lado, otros estudios han destacado (Carrasco et al., 2009; Rothenberg et al., 2019) la naturaleza bidireccional de la relación entre conductas parentales y ajuste psicológico, señalando el efecto directo que tienen los problemas exteriorizados de los hijos sobre las conductas de afecto parentales, por lo tanto, la mayor asociación entre rechazo parental y problemas exteriorizados podría estar mostrando esta realidad bidireccional. En este sentido, es importante recordar que la presencia de problemas interiorizados o exteriorizados puede estar, además, sesgando la percepción del menor sobre las conductas parentales.

Cuando analizamos los efectos parciales del rechazo parental, independientemente del contexto familiar de afecto, sobre los problemas de ajuste de los hijos/as los resultados se muestran consistentes con lo obtenido en el análisis previo de las correlaciones. En este caso y de acuerdo con la segunda hipótesis formulada («el rechazo materno y el paterno tendrán un efecto significativo y negativo sobre el ajuste psicológico de los menores») los resultados proporcionaron un apoyo parcial a la misma. Según nuestros resultados, consistentes con estudios previos (Carrasco et al., 2019; Miranda et al., 2016), la aceptación-rechazo paterna informada por los propios padres (no madres) contribuyen significativa e independiente a los problemas tanto interiorizados como exteriorizados de los hijos y más allá de la fuente informante del desajuste psicológico. En cambio, la aceptación-rechazo materna informada por las madres solo supone una contribución significativa a los problemas exteriorizados informados por los menores. Estos resultados están parcialmente apoyados por estudios previos que indican que las conductas de crianza paternas contribuyen de manera más relevante al ajuste psicológico de hijos e hijas que el de las madres (Hoeve et al., 2009; Miranda et al., 2016; Rothenberg et al., 2019). No obstante, este es un tema controvertido y aún inconcluso, tal y como muestran otros estudios en los que es precisamente el rechazo materno el que emerge frente al paterno como más determinante del desajuste de los hijos/as (Carrasco et al., 2015; Flouri, 2010). Quizás y como se muestra en este trabajo, la fuente informante, tanto de las variables de crianza como del ajuste infantil, pueda ser una condición que incida sobre la contribución relativa de los padres versus las madres al ajuste infantil.

Además de las diferencias halladas en la contribución parcial de los padres a los problemas interiorizados y exteriorizados de los menores según las fuentes informantes, el contexto de afecto familiar percibido por el niño resultó ser un moderador significativo bajo determinadas circunstancias. Tal y como se esperaba en lo formulado en la tercera hipótesis («existirá un efecto moderador del afecto familiar percibido por el/la menor en la contribución del rechazo materno y paterno sobre los problemas exteriorizados e interiorizados de los hijos/as») la percepción subjetiva del contexto afectuoso o no afectuoso por parte del menor moderaba el efecto del rechazo materno sobre los problemas exteriorizados informados por el padre, de tal manera que la relación entre rechazo materno y ajuste psicológico de los menores solo resultaba significativa en el grupo que percibe menos afecto familiar. El efecto del rechazo paterno sobre el ajuste de los hijos/as no resultó significativo en ninguna de las condiciones analizadas, lo que apoya nuestra hipótesis para el rechazo materno, pero no para el paterno. Resultados similares se pueden encontrar en la literatura (Germán et al., 2013), en estudios en los que los efectos del rechazo materno sobre el desajuste se ven amortiguados por la percepción de afecto en el contexto familiar, así como por variables culturales relacionadas con el rol afectivo de la figura materna. Puesto que en nuestra cultura el ambiente familiar afectuoso es normativo, los menores percibirían un ambiente familiar no afectuoso como ilegítimo, lo que potenciaría los efectos del rechazo materno (Lansford et al., 2010). No obstante, McKee y colaboradores (2007) hallaron estos resultados tanto para conductas de crianza materna como paternas. La ausencia de moderación por el contexto familiar de afecto sobre el rechazo paterno informado por el propio padre (pero no materno) en nuestra muestra plantea una incógnita, que junto a la escasez de investigaciones que han incorporado la figura paterna en sus análisis, abre una línea para futuras investigaciones.

Este estudio tiene algunas limitaciones. Con el fin de distinguir las familias con un contexto más afectuoso de aquellas familias con un contexto menos afectuoso se optó por seguir el criterio de investigaciones precedentes (Khaleque et al., 2008; Rohner et al., 2005) y se operacionalizó la variable moderadora, «afecto familiar percibido», en términos de la información aportada por el menor sobre su propia percepción de rechazo. Dado que, nuestra muestra pertenece a población general, en la que la mayor parte de los menores se sienten aceptados (del Barrio et al., 2014), los valores de rechazo percibido por 
los menores participantes eran relativamente bajos, por lo que nuestros resultados no se pueden extrapolar a menores que perciban unos valores de extremos de rechazo. Del mismo modo, el origen de la muestra es española y los hallazgos podrían resultar diferentes en otros contextos culturales. Por otro lado, las asociaciones estadísticas encontradas pueden deberse a la varianza compartida del método (Neyer, 2006). Estudios futuros deberán analizar estas limitaciones y estimar el alcance que tienen sobres los presentes resultados. El estudio futuro de la edad debería igualmente contemplarse. Nuestros datos mostraron una tendencia a percibir más afecto familiar entre los niños/as más pequeños frente a los de mayor edad. Investigaciones precedentes (Rodríguez, del Barrio y Carrasco, 2009; Rosa-Alcazar, Parada-Navas y Rosa-Alcázar, 2014) han hallado el incremento de hostilidad y negligencia parentales percibidas por los adolescentes, quienes informan de un menor apoyo por parte de sus padres a diferencia de lo hallado entre niños/as más pequeños. Esto hace recomendable explorar el efecto moderador de la edad en la relación entre las variables estudiadas. Por otro lado, sería conveniente la replicación de este estudio con muestras en las que los menores perciban un contexto familiar con unos niveles de afecto más bajos.

A pesar de las limitaciones y las lagunas aún no resueltas, es importante destacar la contribución e implicaciones de estos resultados. A pesar de la multitud de trabajos que han señalado la necesidad de investigar en qué grado las distintas fuentes informantes inciden en las relaciones entre unas variables y otras, la literatura al respecto sigue siendo escasa. En este sentido, el presente trabajo ahonda en este campo de conocimiento y remarca la necesidad de tener en cuenta la información de distintos informantes como fuente potencial de variación y moderación de los resultados. De todo ello cabe concluir la necesidad de incluir tanto a los propios menores como a sus padres y madres en la evaluación psicológica, haciendo énfasis en la inclusión sistemática de la figura patena en las investigaciones (Schoppe-Sullivan y Fagan, 2020); y en la conveniencia de considerar la percepción del propio menor sobre el afecto percibido en su contexto familiar cuando se analiza la contribución de la aceptación-rechazo parental en el ajuste psicológico infantil.

\section{Conflictos de interés}

Los autores declaran no tener conflictos de interés.

\section{Referencias}

Achenbach, T. M., Ivanova, M. Y. y Rescorla, L. A. (2017). Empirically based assessment and taxonomy of psychopathology for ages $1 \frac{1}{2}-90+$ years: Developmental, multi-informant, and multicultural findings. Comprehensive Psychiatry, 79, 4-18. https://doi.org/10.1016/j.comppsych. 2017.03.006

Achenbach, T. M. y Rescorla, L. A. (2001). Manual for the ASEBA school-age forms \& profiles: An integrated system of multiinformant assessment. University of Vermont Research Center for Children, Youth, \& Families.

Ali, S., Khaleque, A. y Rohner, R. P. (2015). Pancultural gender differences in the relation between perceived parental acceptance and psychological adjustment of children and adult offspring: A meta-analytic review of worldwide research. Journal of Cross-Cultural Psychology, 46, 1059-1080. https:// doi.org/10.1177/0022022115597754

Berg-Nielsen, T. S., Vika, A. y Dahl, A. A. (2003). When adolescents disagree with their mothers: CBCL-YSR discrepancies related to maternal depression and adolescent self-esteem. Child: Care, Health and Development, 29, 207-213.

Carrasco, M. Á., Delgado, B. y Holgado-Tello, F. P. (2019). Parental acceptance and children's psychological adjustment: The moderating effects of interpersonal power and prestige across age. PLOS ONE, 14, e0215325. https://doi.org/10.1371/ journal.pone. 0215325

Carrasco, M. Á., Holgado-Tello, F. P. y Rodríguez, M. Á. (2015). Intraparental inconsistency: The influence of parenting behaviors on aggression and depression in children. Family Relations, 64, 621-634. https://doi.org/10.1111/fare.12168

Carrasco, M. Á., Holgado-Tello, F. P., Rodríguez, M. Á. y del Barrio, M. V. (2009). Concurrent and across-time relations between mother/father hostility and children's aggression: A longitudinal study. Journal of Family Violence, 24, 213-220. https://doi.org/10.1007/s10896-009-9222-y

Cummings, E. M., Merrilees, C. E. y George, M. W. (2010). Fathers, marriages, and families. En M. E. Lamb (Ed.). The role of the father in child development, (pp. 154-176). John Wiley \& Sons Inc.

de Albéniz-Garrote, G. P., Rubio-Rubio, L. y Medina-Gómez, B. (2018). Papel moderador de los estilos parentales en la relación entre la impulsividad y el consumo de alcohol en una muestra de adolescentes españoles. Revista de Psicopatología y Psicología Clínica, 23(1), 47-57. https://doi.org/10.5944/ rppc.vol.23.num.1.2018.19097

De Los Reyes, A., Lerner, M. D., Keeley, L. M., Weber, R. J., Drabick, D. A. G., Rabinowitz, J. y Goodman, K. L. (2019). Improving interpretability of subjective assessments about psychological phenomena: A review and cross-cultural metaanalysis. Review of General Psychology, 23, 293-319. https:// doi.org/10.1177/1089268019837645

De Los Reyes, A., Ohannessian, C. M. y Racz, S. J. (2019). Discrepancies between adolescent and parent reports about family relationships. Child Development Perspectives, 13, 5358. https://doi.org/10.1111/cdep.12306

del Barrio, M. V., Ramírez-Uclés, I., Romero, C. y Carrasco, M. Á. (2014). Adaptación del Child-PARQ/control: Versiones para el padre y la madre en población infantil y adolescente española. Acción Psicológica, 11, 27-46. https://doi. org/10.5944/ap.11.2.1417

Fisher, R. A. (1925). Applications of «Student's» distribution. Metron, 5, 90-104. 
Flouri, E. (2010). Fathers' behaviors and children's psychopathology. Clinical Psychology Review, 30, 363-369. https://doi.org/10.1016/j.cpr.2010.01.004

Germán, M., Gonzales, N. A., McClain, D. B., Dumka, L. y Millsap, R. (2013). Maternal warmth moderates the link between harsh discipline and later externalizing behaviors for Mexican American adolescents. Parenting, 13, 169-177. https://doi.org/10.1080/15295192.2013.756353

Glicklich, R. (2014). Interpersonal distress in young adulthood: The impact of corporal punishment and perceived parental acceptance and rejection [Tesis doctoral, Long Island University, The Brooklyn Center]. https://search.proquest.com/ docview/1635030465/abstract/D121AC49E7474B25PQ/3

Hoeve, M., Dubas, J. S., Eichelsheim, V. I., van der Laan, P. H., Smeenk, W. y Gerris, J. R. M. (2009). The relationship between parenting and delinquency: A meta-analysis. Journal of Abnormal Child Psychology, 37, 749-775. https://doi. org/10.1007/s10802-009-9310-8

Hunsley, J. y Mash, E. J. (2007). Evidence-based assessment. Annual Review of Clinical Psychology, 3, 29-51. https://doi. org/10.1146/annurev.clinpsy.3.022806.091419

Izquierdo-Sotorrío, E., Holgado-Tello, F. P. y Carrasco, M. Á. (2016). Incremental validity and informant effect from a multi-method perspective: Assessing relations between parental acceptance and children's behavioral problems. Frontiers in Psychology, 7. https://doi.org/10.3389/fpsyg. 2016.00664

Johnston, C. y Murray, C. (2003). Incremental validity in the psychological assessment of children and adolescents. Psychological Assessment, 15, 496-507. https://doi.org/10.1037/ 1040-3590.15.4.496

Kazdin, A. E. (2005). Evidence-based assessment for children and adolescents: Issues in measurement development and clinical application. Journal of Clinical Child \& Adolescent Psychology, 34, 548-558. https://doi.org/10.1207/s15374424jccp 3403_10

Khaleque, A. (2018). Worldwide implications of parental love and lack of love on children and adults psychological adjustment and maladjustment: Meta-analytic evidence. Journal of Mental Disorders and Treatment, 4, 150. https://doi.org/10. 4172/2471-271X.1000150

Khaleque, A., Rohner, R. P. y Nahar, Z. (2008). Agreement between children's and mothers' perceptions of maternal acceptance-rejection: A comparative study of mothers and children in Bangladesh and Bangladeshi immigrant families in the United States. En F. Erkman (Ed.), Acceptance: The essence of peace (pp. 175-185). Turkish Psychology Association.

Lansford, J. E., Malone, P. S., Dodge, K. A., Chang, L., Chaudhary, N., Tapanya, S., Oburu, P. y Deater-Deckard, K. (2010). Children's perceptions of maternal hostility as a mediator of the link between discipline and children's adjustment in four countries. International Journal of Behavioral Development, 34, 452-461. https://doi.org/10.1177/0165025409354933

McKee, L., Roland, E., Coffelt, N., Olson, A. L., Forehand, R., Massari, C., Jones, D., Gaffney, C. A. y Zens, M. S. (2007). Harsh discipline and child problem behaviors: The roles of positive parenting and gender. Journal of Family Violence, 22, 187-196. https://doi.org/10.1007/s10896-007-9070-6
Meunier, J. C., Bisceglia, R. y Jenkins, J. M. (2012). Differential parenting and children's behavioral problems: Curvilinear associations and mother-father combined effects. Developmental Psychology, 48, 987-1002. https://doi.org/ 10.1037/a0026321

Miranda, M. C., Affuso, G., Esposito, C. y Bacchini, D. (2016). Parental acceptance-rejection and adolescent maladjustment: Mothers' and fathers' combined roles. Journal of Child and Family Studies; New York, 25, 1352-1362. http://dx.doi. org/10.1007/s10826-015-0305-5

Neyer, F. (2006). Informant assessment. En M. Eid y E. E. Diener (Eds.), Handbook of multimethod measurement in psychology (pp. 43-59). American Psychological Association.

Ordóñez-López, A. (2015). Ajuste psicológico en la infancia: aspectos emocionales y variables asociadas [Tesis doctoral, Universitat de Valencia]. https://core.ac.uk/download/pdf/ 71043910.pdf

Putnick, D. L., Bornstein, M. H., Lansford, J. E., Malone, P. S., Pastorelli, C., Skinner, A. T., Sorbring, E., Tapanya, S., Tirado, L. M. U., Zelli, A., Alampay, L. P., Al-Hassan, S. M., Bacchini, D., Bombi, A. S., Chang, L., Deater-Deckard, K., Di Giunta, L., Dodge, K. A. y Oburu, P. (2015). Perceived mother and father acceptance-rejection predict four unique aspects of child adjustment across nine countries. Journal of Child Psychology and Psychiatry, and Allied Disciplines, 56, 923932. https://doi.org/10.1111/jcpp.12366

Richters, J. E. (1992). Depressed mothers as informants about their children: A critical review of the evidence for distortion. Psychological Bulletin, 112, 485-499.

Rodríguez, M. M. (2017). Análisis de la implicación del padre en el ajuste psicológico de los hijos: Mediación de la aceptaciónrechazo parental percibida [Tesis doctoral, UNED]. http://espacio.uned.es/fez/view/tesisuned:Psicologia-Mmrodriguez

Rodríguez, M. A., del Barrio, M. V. y Carrasco, M. Á. (2009). ¿Cómo perciben los hijos la crianza materna y paterna? Diferencias por edad y sexo. Escritos de Psicología, 2, 10-18.

Rohner, R. P. (2004). The parental «acceptance-rejection syndrome»: Universal correlates of perceived rejection. American Psychologist, 59, 830-840. https://doi.org/10.1037/ 0003-066X.59.8.830

Rohner, R. P. (2016). Introduction to interpersonal acceptancerejection theory (IPARTheory) and evidence. Online Readings in Psychology and Culture, 6, 1-40. https://doi.org/10.9707/ 2307-0919.1055

Rohner, R. P. y Ali, S. (2016). Parental acceptance-Rejection Questionnaire (PARQ). En V. Zeigler-Hill y T. K. Shackelford (Eds.), Encyclopedia of personality and individual differences (pp. 1-4). Springer International Publishing. https://doi. org/10.1007/978-3-319-28099-8_56-1

Rohner, R. P., Khaleque, A., Riaz, M. N., Khan, U., Sadeque, S. y Laukkala, H. (2005). Agreement between children's and mothers' perceptions of maternal acceptance and rejection: A Comparative Study in Finland and Pakistan. Ethos, 33, 367377. https://doi.org/10.1525/eth.2005.33.3.367

Rosa-Alcazar, A. I., Parada-Navas, J. L. y Rosa-Alcázar, A. (2014). Síntomas psicopatológicos en adolescentes españoles: Relación con los estilos parentales percibidos y la autoestima. Anales de Psicología, 30, 133-142. https://doi.org/10.6018/ analesps.30.1.165371 
Rothenberg, W. A., Lansford, J. E., Alampay, L. P., Al-Hassan, S. M., Bacchini, D., Bornstein, M. H., Chang, L., DeaterDeckard, K., Giunta, L. D., Dodge, K. A., Malone, P. S., Oburu, P., Pastorelli, C., Skinner, A. T., Sorbring, E., Steinberg, L., Tapanya, S., Tirado, L. M. U. y Yotanyamaneewong, S. (2019). Examining effects of mother and father warmth and control on child externalizing and internalizing problems from age 8 to 13 in nine countries. Development and Psychopathology, 1-25. https://doi.org/10.1017/S0954579419001214

Sánchez-Sánchez, F., Fernández-Pinto, I., Santamaría, P., Carrasco, M. Á. y del Barrio, M. V. (2016). SENA, Sistema de
Evaluación de Niños y Adolescentes: Proceso de desarrollo y evidencias de fiabilidad y validez. Revista de Psicología Clínica con Niños y Adolescentes, 3, 23-34.

Schoppe-Sullivan, S. J. y Fagan, J. (2020). The evolution of fathering research in the 21 st century: Persistent challenges, New Directions. Journal of Marriage and Family, 82(1), 175197. https://doi.org/10.1111/jomf.12645

van Doorn, M. M. E. M., Bodden, D., Jansen, M., Rapee, R. M. y Granic, I. (2018). Linking mother-child discrepancies to behavioral observations of children's anxiety. Child \& Youth Care Forum, 47, 481-498. https://doi.org/10.1007/s10566-018-9441-6 